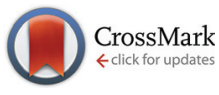

Cite this: Dalton Trans., 2015, 44 13480

Received 24th December 2014, Accepted 25th June 2015

DOI: $10.1039 / \mathrm{c} 4 \mathrm{dt} 03980 \mathrm{~g}$

www.rsc.org/dalton

\section{Field and dilution effects on the magnetic relaxation behaviours of a 1D dysprosium(III)- carboxylate chain built from chiral ligands $\uparrow$}

\author{
Tian Han, ${ }^{a}$ Ji-Dong Leng, ${ }^{a}$ You-Song Ding, ${ }^{a}$ Yanyan Wang, ${ }^{a}$ Zhiping Zheng, and \\ Yan-Zhen Zheng*a
}

\begin{abstract}
A one-dimensional dysprosium(III)-carboxylate chain in which the Dy ${ }^{\text {III }}$ ions sit in a pseudo $D_{2 \mathrm{~d}}$-symmetry environment is synthesized and shows different slow magnetic relaxation behaviours depending on the field and dilution effects. Besides, the chiral ligand introduces the additional functions of the Cotton effect and polarization for this compound.
\end{abstract}

Since the discovery of mononuclear lanthanide complexes functioning as single molecule magnets (SMMs), ${ }^{1}$ lanthanide ions with large orbital momentum and strong magnetic anisotropy have been broadly used in developing new generation molecule-based magnetic information storage materials. ${ }^{2}$ Amongst these molecular materials dysprosium(III)-based complexes are particularly popular due to the inherent strong spin-orbital coupling effect and hence very high magnetic anisotropy of the ${ }^{6} \mathrm{H}_{15 / 2}$ state with ground state Kramers doublet. ${ }^{3-5}$ The magnetic anisotropy of the Dy ${ }^{\mathrm{III}}$ ions is significantly affected by the coordination geometry and the strength of the ligand field, which governs the barrier height of the $\mathrm{Dy}^{\mathrm{III}}$ ions for magnetization-reversal. ${ }^{6}$

Other than the dominated single-ion behaviour the intriguing roles of magnetic exchange-coupling and dipole-dipole interactions between the $4 \mathrm{f}$ ions are complicated. As shown by several groups, on the one hand, weak magnetic interaction between the $4 \mathrm{f}$ ions allows the quantum tunneling effect that mitigates the full potential of magnetic blocking, ${ }^{3 i, 7}$ while on the other hand, strong magnetic exchange enhances the slow magnetic relaxation. ${ }^{3 h, 8}$ Hitherto, understanding the nature of the magnetic relaxation in Dy III-based mononuclear SMMs remains challenging, especially when the lanthanide ions are chemically linked.

${ }^{a}$ Center for Applied Chemical Research, Frontier Institute of Science and Technology, and College of Science, Xi'an Jiaotong University, Xi'an 710054, China.

E-mail: zheng:yanzhen@mail.xjtu.edu.cn

${ }^{b}$ Department of Chemistry, The University of Arizona, Tucson, Arizona 85721, USA $\dagger$ Electronic supplementary information (ESI) available: Complete experimental detail, crystal and refinement details, supporting figures and additional magnetic data. CCDC 1027340 and 1030432. For ESI and crystallographic data in CIF or other electronic format see DOI: 10.1039/c4dt03980g
For structurally one-dimensional (1D) Dy ${ }^{\mathrm{III}}$-systems, dynamic magnetic behaviour may be observed. ${ }^{4,5}$ However, due to the subtle magnetic exchange-coupling between the lanthanide centres, whether the relaxation is underpinning by the single-ion or single-chain magnetic origin is debatable. To thoroughly understand the magnetic dynamics of these 1D chain systems magnetic dilution is thus essential. ${ }^{9}$ Uniquely, magnetic dilution in molecular systems can be successfully accomplished by the doping of chemically-identical diamagnetic metal ions (i.e. $\mathrm{Y}^{\mathrm{III}}$ ), which was highlighted in some mononuclear and polynuclear lanthanide molecular magnets. ${ }^{1,3 e, i, 7}$ Moreover, the introduction of chirality might bring multi-functionality to the system such as ferroelectricity and magneto-optical coupling effect. ${ }^{10}$

Pursuing these clues, we report herein the syntheses, structures and magnetic properties of a 1D dysprosium(III)-carboxylate compound $\left\{\left[\mathrm{Dy}(\mathrm{L})_{3}\left(\mathrm{H}_{2} \mathrm{O}\right)\right] \cdot 5 \mathrm{H}_{2} \mathrm{O}\right\}_{n}(\mathbf{1})$ and its $50 \%$ diluted sample $\left\{\left[\mathrm{Dy}_{0.5} \mathrm{Y}_{0.5}(\mathrm{~L})_{3}\left(\mathrm{H}_{2} \mathrm{O}\right)\right] \cdot 5 \mathrm{H}_{2} \mathrm{O}\right\}_{n}$ (2) (HL = D-(-)-quinic acid). Slow relaxation of magnetization can be modulated as suppressing the quantum tunneling effect differently by field and/or dilution.

The reaction of D-(-)-quinic acid with $\mathrm{Dy}\left(\mathrm{NO}_{3}\right)_{3} \cdot 6 \mathrm{H}_{2} \mathrm{O}$ in water-acetonitrile, in the presence of $\mathrm{Et}_{3} \mathrm{~N}$, affords well shaped colorless needle-like crystals. A single-crystal X-ray structural study at $150 \mathrm{~K}$ indicates that $\mathbf{1}$ belongs to the monoclinic system with the $P 2_{1}$ space group. Crystallographic data, structure refinement, selected bonds and angles are listed in Tables S1 and S2 (ESI $\dagger$ ). The Dy ${ }^{\text {III }}$ ion is eight coordinated, surrounded by oxygen atoms, six of which are from three chelating ligands, one from $\mu_{2}$ bridging ligands and one from coordinated water molecules (Fig. 1 and S1, ESI†). In the literature, the geometries of eight-coordinate Dy ${ }^{\text {III }}$ ions are mostly taking symmetries such as the $D_{2 \mathrm{~d}}$ - dodecahedron (DD), $C_{2 \mathrm{v}}$ bicapped trigonal prism (TP) and $D_{4 \mathrm{~d}}$ - square antiprism (SAP). The continuous symmetry measure (CSM) method for $\mathbf{1}$ indicates that the DD geometry is the closest one (calcd values: $\left.D_{2 \mathrm{~d}}, 0.961 ; D_{4 \mathrm{~d}}, 1.716 ; C_{2 \mathrm{v}}, 2.289\right) .{ }^{11}$ Further study using the semi-quantitative method of polytopal analysis according to the relevant dihedral angles in Table S3 (ESI $\dagger$ ) confirms that 


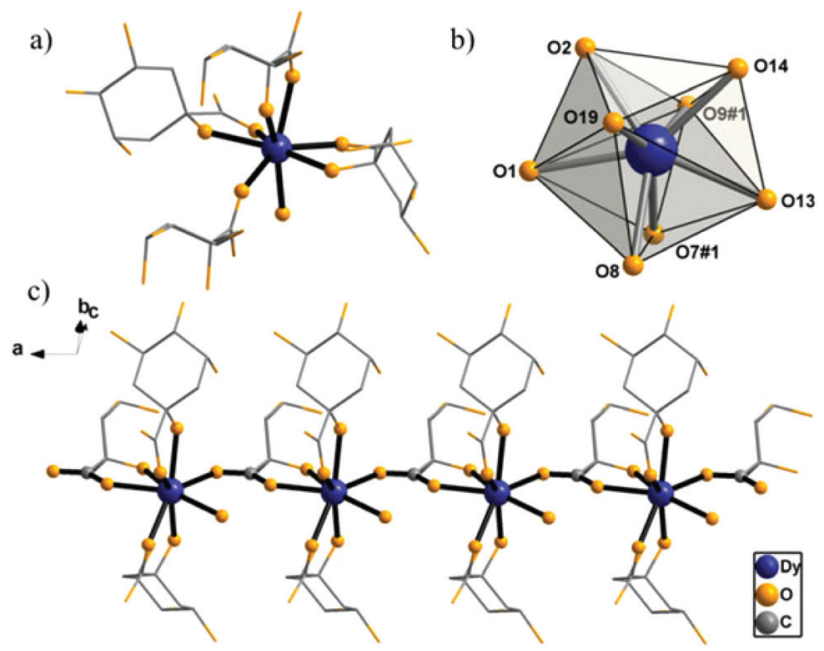

Fig. 1 (a) Local structure of 1; (b) the coordination polyhedron of Dy ${ }^{\prime \prime \prime}$ in 1; (c) 1D chain structure of 1 . Symmetry code: $\# 1 x+1, y, z$.

the Dy ${ }^{\mathrm{III}}$ ion in 1 has the distorted $D_{2 \mathrm{~d}}$ local symmetry. ${ }^{12}$ The neighboring Dy ${ }^{\mathrm{III}}$ ions are singly bridged by $\mu-\eta^{1}: \eta^{1}$ carboxylic groups in the syn-anti mode to form a one-dimensional infinite chain, resulting in the Dy $\cdots$ Dy distance of $6.085 \AA$ A. Adjacent chains are further expanded to a $3 \mathrm{D}$ supramolecular architecture via strong hydrogen bonds between the water molecules and the quinate ligands with the $\mathrm{O} \cdots \mathrm{O}$ distances ranging from 2.608(3) to 3.231(4) $\AA$ (Fig. S2, ESI $\dagger$ ). The nearest interchain Dy $\cdots$ Dy separation is 10.5635(4) ̊.

Meanwhile, the $\mathrm{Y}^{\mathrm{III}}$ doped sample $\left\{\left[\mathrm{Dy}_{0.5} \mathrm{Y}_{0.5}(\mathrm{~L})_{3}\left(\mathrm{H}_{2} \mathrm{O}\right)\right] \cdot 5 \mathrm{H}_{2} \mathrm{O}\right\}_{n}$ (2) is prepared to modulate the magnetic properties. Inductively coupled plasma (ICP) measurement is thus necessary to study the composition precisely. Thus the exact ratio for $\mathrm{Dy}^{\mathrm{III}}$ : $\mathrm{Y}^{\mathrm{III}}$ is $1.04: 1.00$ which is perfectly consistent with the starting stoichiometry in the synthesis. The single-crystal structural data indicate that compound $\mathbf{2}$ is isostructural to $\mathbf{1}$ with indistinguishable metal centres (Table S4, ESI†). The good agreement of powder X-ray diffraction patterns between $\mathbf{1}$ and $\mathbf{2}$ further confirms the phase purity of the bulk materials (Fig. S3, ESI†े).

Dc magnetic susceptibility data of $\mathbf{1}$ and $\mathbf{2}$ are measured in an applied field of $2 \mathrm{kOe}$ and in the temperature range of 2-300 K (Fig. 2). At $300 \mathrm{~K}$, the $\chi_{\mathrm{M}} T$ product of 1 is $14.54 \mathrm{~cm}^{3} \mathrm{~K}$ $\mathrm{mol}^{-1}$, close to $14.17 \mathrm{~cm}^{3} \mathrm{~K} \mathrm{~mol}^{-1}$ expected for one $\mathrm{Dy}{ }^{\mathrm{III}}$ ion $(S=5 / 2, L=5, J=15 / 2$ and $g=4 / 3)$. Upon cooling, the $\chi_{\mathrm{M}} T$ products remained constant until $30 \mathrm{~K}$, and then decreased sharply to the minimum of $10.02 \mathrm{~cm}^{3} \mathrm{~K} \mathrm{~mol}^{-1}$ at $2 \mathrm{~K}$. For 2, the $\chi_{\mathrm{M}} T$ product $14.39 \mathrm{~cm}^{3} \mathrm{~K} \mathrm{~mol}^{-1}$ at $300 \mathrm{~K}$ accords well with the expected value. Upon cooling, similar behaviour of the temperature-dependent $\chi_{\mathrm{M}} T$ products is observed, but the lowest value $9.35 \mathrm{~cm}^{3} \mathrm{~K} \mathrm{~mol}^{-1}$ at $2 \mathrm{~K}$ is slightly smaller than the undoped sample. Because the coordination environments of the Dy ${ }^{\mathrm{III}}$ ions remain unchanged, comparison of magnetic data of $\mathbf{2}$ with that of $\mathbf{1}$ could reveal that the ferromagnetic interaction in the system is weakened by the dilution method.

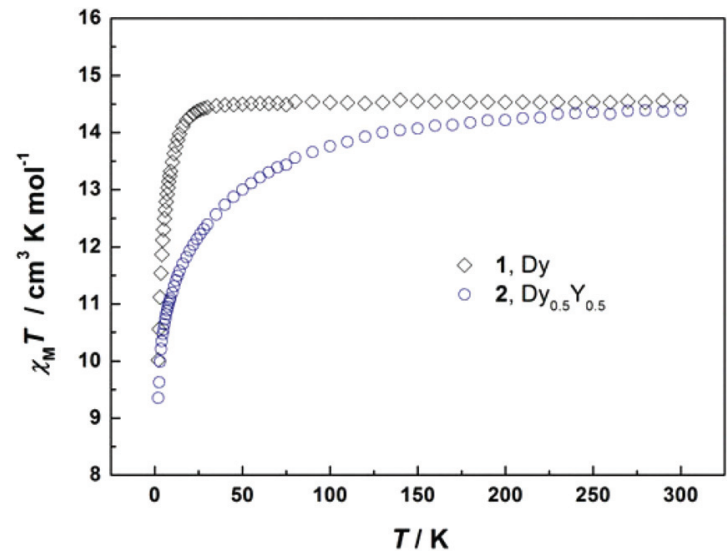

Fig. $2 \chi_{M} T$ as a function of temperature in an applied field of $2 \mathrm{kOe}$ for 1 and 2. The $\chi_{M} T$ curve of 2 has been rescaled for one Dy"l ion.

The field dependences of magnetization for both compounds have been determined at low temperatures. Upon applying the magnetic field, magnetizations increase up to maximum values of 7.18 and $6.03 \mathrm{~N} \beta$ at $70 \mathrm{kOe}$ and $2 \mathrm{~K}$ for 1 and 2, respectively, without saturation. This may be due to the large magneto-anisotropy and/or the low lying excited states of the Dy ${ }^{I I I}$ ions, as also proved by the non-superposition on $M / N \beta$ versus $H T^{-1}$ plots at various temperatures in 1 (Fig. S4, ESI $\dagger$ ).

To further explore the dynamics of magnetization, ac magnetic susceptibilities as functions of both temperature and frequency are studied for both samples. For $\mathbf{1}$, a significant frequency dependence of ac signals can be observed in zero dc field, indicating slow relaxation of magnetization, but unfortunately, no maximum of peak is found in both the in-phase $\left(\chi^{\prime}\right)$ and out-of-phase $\left(\chi^{\prime \prime}\right)$ ac signals, which is possible due to the tunneling of the magnetization (QTM) (Fig. 3a). As the application of an external magnetic field can suppress the QTM effect, ${ }^{3,7,13}$ subsequent ac measurements were taken under various dc fields (Fig. S5, ESI $\dagger$ ).

At $H_{\mathrm{dc}}=500$ Oe obvious peaks of the $\chi^{\prime \prime}$ data can be detected, and move to higher temperature with increasing frequency, clearly showing the frequency dependent behavior (Fig. S6, ESI $\dagger$ ). A peak tail at low temperatures is indicative of QTM often reported in lanthanide SMMs. ${ }^{1,3,6-9,14}$ The ac susceptibility as a function of frequency with varying temperatures reveals two independent relaxation domains. Relaxation in domain \#1 shifts from low frequency to high frequency upon warming, while relaxation domain \#2 involves high frequencies with temperature independent feature (Fig. S7, ESI $\dagger$ ). When the relaxation time $(\tau)$ is plotted as $\ln \tau$ vs. 1/T (Fig. 3b), one thermally activated regime (pathway A) and a gradual crossover to the temperature independent relaxation regime (pathway B, $\tau=76 \mathrm{~ms}$ at $2.0 \mathrm{~K}$, indicative of a QTM process) in domain $\# 1$, and relaxation at $\tau \approx 0.3 \mathrm{~ms}$ in domain $\# 2$ (pathway C) are obtained. ${ }^{15}$ Analysis of the data for the thermally activated regime (A) using the Arrhenius law gives a preexponential factor of $\tau_{0}=1.6 \times 10^{-10} \mathrm{~s}$ and an effective energy 

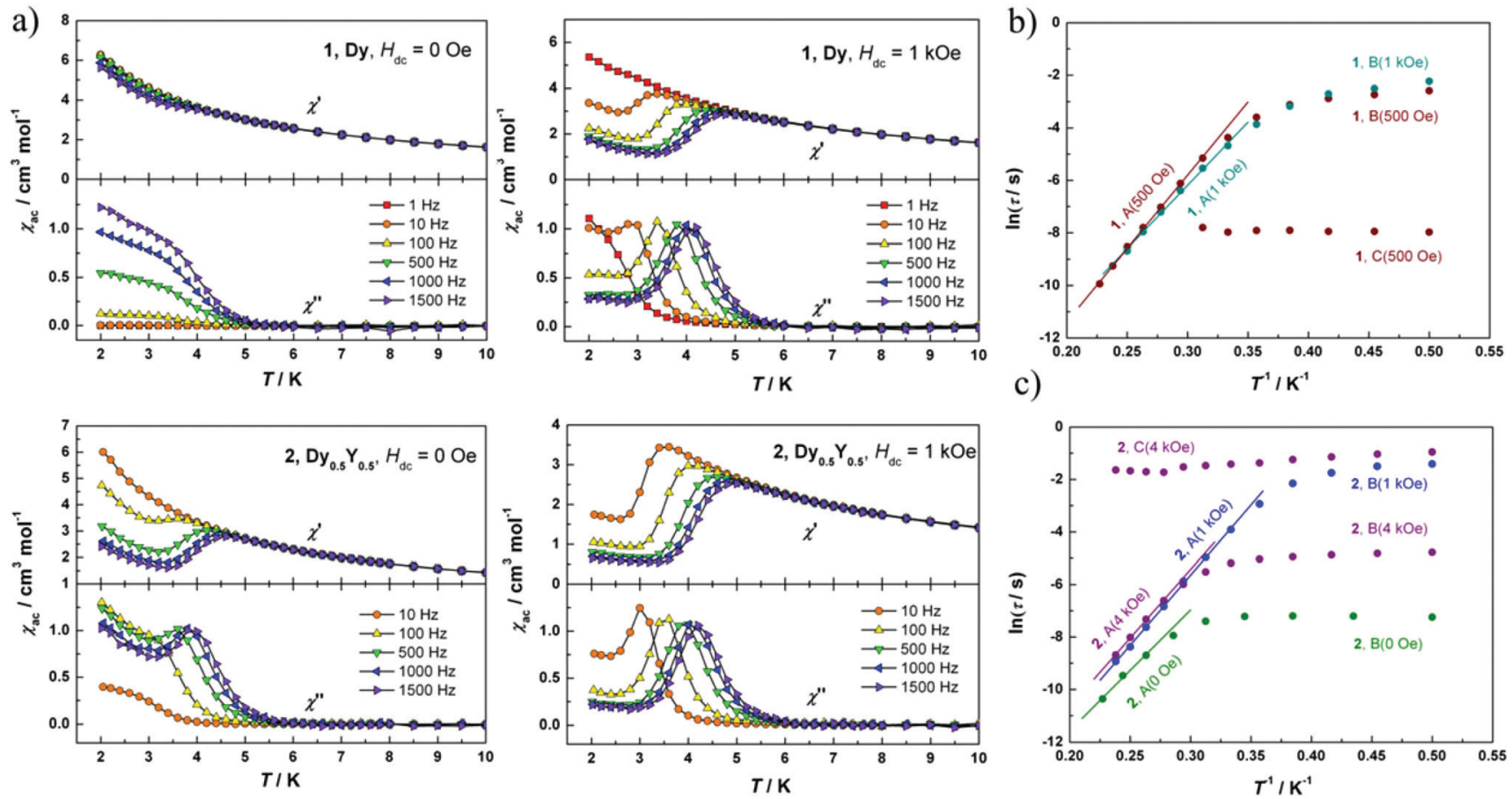

Fig. 3 (a) Temperature dependence of the in-phase and out-of-phase ac susceptibility for 1 and 2 under 0 or 1 kOe dc field. (b) Plots of ln $(\tau)$ vs. $1 / T$ for 1 at $H_{\mathrm{dc}}=500 \mathrm{Oe}$ and $1 \mathrm{kOe}$. (c) Plots of $\ln (\tau)$ vs. $1 / T$ for 2 at $H_{\mathrm{dc}}=0,1 \mathrm{kOe}$ and $4 \mathrm{kOe}$. The solid lines represent Arrhenius fits of the frequencydependent data.

gap of $U_{\text {eff }}=55.8 \mathrm{~K}$. Cole-Cole plots suggest that relaxation domains \#1 and \#2 are both well-defined at low temperatures, and begin to merge above $3.0 \mathrm{~K}$ as the peak of $\chi^{\prime \prime}$ in domain \#1 shifts to high frequency (Fig. S8, ESI $\dagger$ ). At higher temperature, the thermally activated pathway $\mathrm{A}$ is dominant.

On increasing the dc field to $1 \mathrm{kOe}$, the process in relaxation domain \#2 is eliminated, leaving only one uniform relaxation domain (Fig. S5, ESI $\dagger$ ). The ac susceptibilities as functions of both temperature and frequency at $1 \mathrm{kOe}$ confirm the remaining processes in relaxation domain \#1 (Fig. 3a and $\mathrm{S} 9, \mathrm{ESI} \dagger$ ). As the temperature decreases from 4.0 to $3.0 \mathrm{~K}$, the maximum in $\chi^{\prime \prime}$ moves gradually to lower frequency, falling into an Arrhenius-like behavior region (pathway $\mathrm{A}, \tau_{0}=1.1 \times$ $10^{-9} \mathrm{~s}$ and $U_{\text {eff }}=48.2 \mathrm{~K}$ ). Below $3.0 \mathrm{~K}$, the relaxation is dominated by a quantum tunneling process (pathway B, $\tau=107 \mathrm{~ms}$ at $2.0 \mathrm{~K}$ ) (Fig. 3b). Cole-Cole plots at different temperatures show one semicircle (Fig. S10, ESI $\dagger$ ). The ac measurements indicate that one of the relaxation processes at high frequencies with temperature independent feature can be suppressed by applying a static magnetic field.

Slow magnetic relaxation in a ferromagnetic 1D dysprosium chain through double syn-anti carboxylic groups originating from the single-ion behaviour of $\mathrm{Dy}^{\mathrm{III}}$ has been evidenced by the group of Gao, ${ }^{9}$ in which the closest Dy...Dy distance is $1 \AA$ shorter than that in $\mathbf{1}$ bridged by the single syn-anti carboxylic group. Weaker intrachain exchange coupling of $\mathbf{1}$ further clarifies the domination of single-ion anisotropy in thermal relaxation. It is well known that magnetic interactions with the neighbouring motif can greatly influence the quantum tunneling of magnetization in discrete molecules, ${ }^{3,6-8,16}$ but the doping effect in the 1D lanthanide chain is still poorly understood. ${ }^{9}$

Herein we study the ac magnetic properties of doped sample 2 , with $50 \%$ concentration of the dopant ion to modulate the relaxations. In the plot of $\chi^{\prime \prime}$ data versus $v$ at $2 \mathrm{~K}$ for 2 under various dc fields, the peak with maximum shifts from $162 \mathrm{~Hz}$ at 0 Oe to $0.56 \mathrm{~Hz}$ at $1 \mathrm{kOe}$ dc field (Fig. S11, ESI $\dagger$ ). Surprisingly, when further increasing the dc field, a broad peak shows up and then separates into two sets, one of which resides at about $0.5 \mathrm{~Hz}$, and the other displays strong field dependence with increasing frequency toward higher field.

As shown in Fig. 3a and S12 (ESI $\dagger$ ), Y(III) diluted sample 2 exhibits significant temperature and frequency dependent behaviour with a peak maximum which is absent in $\mathbf{1}$ in a zero dc field. Furthermore, the tail in the $\chi^{\prime \prime}(T)$ plot below $3.0 \mathrm{~K}$ shows strong frequency dependence with increasing intensity toward lower frequency (except $10 \mathrm{~Hz}$ ). This indicates that the QTM effect, albeit remains, is efficiently reduced by dilution. Temperature independent peaks signaling the quantum tunneling region (pathway B) can be observed, ranging from $2.0 \mathrm{~K}$ to $3.2 \mathrm{~K}$, with relaxation time of about $0.7 \mathrm{~ms}$, which is much shorter than the one in $\mathbf{1}$ at applied dc fields. At higher temperatures, the relaxation obeys the Arrhenius law (pathway A), affording $\tau_{0}=9.4 \times 10^{-10} \mathrm{~s}$ and $U_{\text {eff }}=46.1 \mathrm{~K}$ (Fig. 3c). Semicircle Cole-Cole plots suggest that the relaxation domain \#1 is present (Fig. S13, ESI†). 
With an external field of $1 \mathrm{kOe}$, the single peak remains, and the tail in the $\chi^{\prime \prime}(T)$ plot almost disappears at low temperature, indicating a more efficient suppression of QTM (Fig. 3a and S14, ESI $\dagger$ ). The temperature dependence of the relaxation time in the range of 3.0-4.2 $\mathrm{K}$ follows an activated Arrhenius law (pathway A), giving $\tau_{0}=3.8 \times 10^{-10} \mathrm{~s}$ and $U_{\text {eff }}=53.5 \mathrm{~K}$ (Fig. 3c). Deviation from linearity due to the quantum tunneling process (pathway B, $\tau=244 \mathrm{~ms}$ at $2.0 \mathrm{~K}$ ) is observed below $3.0 \mathrm{~K}$. The Cole-Cole plots imply a single relaxation domain (Fig. S15, ESI†).

At 4 kOe, two independent relaxation domains, one at higher frequencies $(3-1500 \mathrm{~Hz}$, relaxation domain \#1) and the other at lower frequencies $(0.1-3 \mathrm{~Hz}$, relaxation domain \#2) appear (Fig. S16 and S17, ESI $\dagger$ ). ${ }^{15}$ Relaxation domain \#1 involves a thermally activated process (pathway A) with $\tau_{0}=$ $6.8 \times 10^{-10} \mathrm{~s}$ and $U_{\text {eff }}=52.3 \mathrm{~K}$ and a temperature independent regime (pathway B, $\tau=8 \mathrm{~ms}$ at $2.0 \mathrm{~K}$ ) (Fig. 3c). Relaxation domain \#2 shows relatively little temperature dependence and field dependence (pathway C). Its relaxation time ( $c a .300 \mathrm{~ms}$ ) is 3 orders of magnitude slower than that of domain $\# 1$, and there is no asymmetry in the Cole-Cole plots for this process (Fig. S18, ESI†े).

Taken together, field and dilution effects result in various degrees of reduction in the quantum tunneling (Fig. S19, ESI $\dagger$ ). Interestingly, multiple relaxation modes are observed and accessible through the application of dc fields, as reported in mononuclear SMMs. ${ }^{15}$ However, due to the absence of peak maximum in ac measurements for the 5\% Dy sample, further experiments and theoretical calculations to investigate the detailed impact of dilution on the dynamic properties are necessary.

Given that 1 crystallises in a chiral space group $P 2_{1}$ at room temperature (Table S5, ESI $\dagger$ ) while it belongs to a point group of $C_{2}$ falling into one of the 10 polar point groups, its optical and ferroelectric properties were investigated at room temperature. As shown in Fig. 4, both the circular dichroism (CD) spectra for the ligand in an aqueous solution and compound 1 in the solid state exhibit a strong positive Cotton effect at

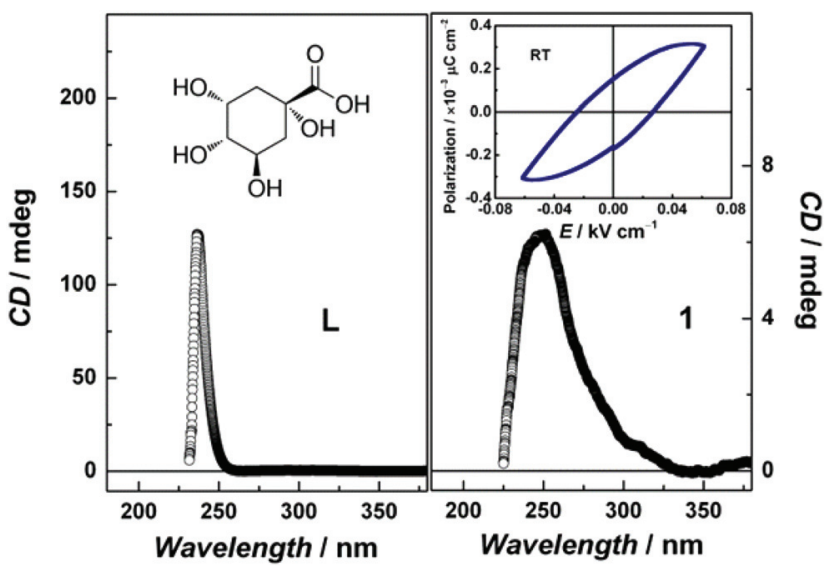

Fig. 4 Circular dichroism spectra for the D-(-)-quinic acid in an aqueous solution (left) and 1 in the solid state (right). Inset: electric hysteresis loop of 1 at room temperature. $\sim 240 \mathrm{~nm}$, denoting significant dichroism associated with the homochiral ligand. An open electric field dependent polarization loop can be clearly, albeit with small remnants, observed for the powder pellet sample of $\mathbf{1}$ (inset Fig. 4), showing the potential ferroelectric behavior.

In summary, a novel enantiomer-pure 1D dysprosium chain has been assembled from a chiral carboxylate ligand. The Dy ${ }^{\text {III }}$ ion in 1 sits in a distorted $D_{2 \mathrm{~d}}$ symmetry, and is bridged by the single syn-anti carboxylic group to form an infinite chain, which shows very weak ferromagnetic interaction and slow relaxation of the magnetization. By the application of a magnetic field and/or dilution, the quantum tunneling is suppressed in various degrees, thereby suggesting the dominant single-ion origin of the slow magnetic relaxation rather than the single-chain dynamics. Thus, a symbol $\mathrm{M}^{0} \mathrm{U}^{0} \mathrm{~S}^{1}$ is presumably appropriate to describe such systems. ${ }^{17}$ Our comparative investigations confirm the plausible fine-tuning of QTM via dilution, which mainly alternates the magnetic interactions between individual metal centres. Moreover, the presence of small remnant in the electric-polarization measurement may bring multiferroic properties to such molecule-based materials.

This work was supported by 973 projects (2012CB619401 and 2012CB619402), NSFC (21201137, 21473129 and IRT13034), the China Postdoctoral Science Foundation (2014M552425), the "National 1000-Plan" program and the Fundamental Research Funds for the Central Universities. This work was also partially supported by the US National Science Foundation (Grant CHE-1152609). We also thank the Nankai University for ferroelectric measurements.

\section{Notes and references}

1 N. Ishikawa, M. Sugita, T. Ishikawa, S. Y. Koshihara and Y. Kaizu, J. Am. Chem. Soc., 2003, 125, 8694.

2 D. N. Woodruff, R. E. P. Winpenny and R. A. Layfield, Chem. Rev., 2013, 113, 5110.

3 (a) P. Zhang, Y. N. Guo and J. K. Tang, Coord. Chem. Rev., 2013, 257, 1728; (b) Y. N. Guo, G. F. Xu, Y. Guo and J. K. Tang, Dalton Trans., 2011, 40, 9953; (c) J. K. Tang, I. Hewitt, N. T. Madhu, G. Chastanet, W. Wernsdorfer, C. E. Anson, C. Benelli, R. Sessoli and A. K. Powell, Angew. Chem., Int. Ed., 2006, 45, 1729; (d) P. H. Lin, T. J. Burchell, R. Clérac and M. Murugesu, Angew. Chem., Int. Ed., 2008, 47, 8848; (e) S. D. Jiang, B. W. Wang, G. Su, Z. M. Wang and S. Gao, Angew. Chem., Int. Ed., 2010, 49, 7448; (f) Y. N. Guo, G. F. Xu, P. Gamez, L. Zhao, S. Y. Lin, R. Deng, J. K. Tang and H. J. Zhang, J. Am. Chem. Soc., 2010, 132, 8538; (g) R. J. Blagg, C. A. Muryn, E. J. L. McInnes, F. Tuna and R. E. P. Winpenny, Angew. Chem., Int. Ed., 2011, 50, 6530; (h) J. D. Rinehart, M. Fang, W. J. Evans and J. R. Long, Nat. Chem., 2011, 3, 538; (i) R. J. Blagg, L. Ungur, F. Tuna, J. Speak, P. Comar, D. Collison, W. Wernsdorfer, E. J. L. McInnes, L. F. Chibotaru and R. E. P. Winpenny, Nat. Chem., 2013, 5, 673; (j) E. M. Fatila, M. Rouzières, 
M. C. Jennings, A. J. Lough, R. Clérac and K. E. Preuss, J. Am. Chem. Soc., 2013, 135, 9596; (k) J. Long, F. Habib, P. H. Lin, I. Korobkov, G. Enright, L. Ungur, W. Wernsdorfer, L. F. Chibotaru and M. Murugesu, J. Am. Chem. Soc., 2011, 133, 5319.

4 (a) Y. Z. Zheng, Y. H. Lan, W. Wernsdorfer, C. E. Anson and A. K. Powell, Chem. - Eur. J., 2009, 15, 12566; (b) I. Ratera and J. Veciana, Chem. Soc. Rev., 2012, 41, 303; (c) L. Bogani, C. Sangregorio, R. Sessoli and D. Gatteschi, Angew. Chem., Int. Ed., 2005, 44, 5817.

5 (a) J. Jung, F. L. Natur, O. Cador, F. Pointillart, G. Calvez, C. Daiguebonne, O. Guillou, T. Guizouarn, B. L. Guennic and K. Bernot, Chem. Commun., 2014, 50, 13346; (b) Q. Chen, Y. S. Meng, Y. Q. Zhang, S. D. Jiang, H. L. Sun and S. Gao, Chem. Commun., 2014, 50, 10434; (c) E. Bartolomé, J. Bartolomé, S. Melnic, D. Prodius, S. Shova, A. Arauzo, J. Luzón, F. Luis and C. Turta, Dalton Trans., 2013, 42, 10153; (d) S. Liu, L. L. Li, H. Li, H. L. Gao, J. Z. Cui and P. Cheng, Dalton Trans., 2015, 44, 6169.

6 (a) J. L. Liu, Y. C. Chen, Y. Z. Zheng, W. Q. Lin, L. Ungur, W. Wernsdorfer, L. F. Chibotaru and M. L. Tong, Chem. Sci., 2013, 4, 3310; (b) B. Na, X. J. Zhang, W. Shi, Y. Q. Zhang, B. W. Wang, C. Gao, S. Gao and P. Cheng, Chem. - Eur. J., 2014, 20, 15975.

7 F. Habib, P. H. Lin, J. Long, I. Korobkov, W. Wernsdorfer and M. Murugesu, J. Am. Chem. Soc., 2011, 133, 8830.

8 (a) J. D. Rinehart, M. Fang, W. J. Evans and J. R. Long, J. Am. Chem. Soc., 2011, 133, 14236; (b) Y. N. Guo, G. F. Xu, W. Wernsdorfer, L. Ungur, Y. Guo, J. K. Tang, H. J. Zhang, L. F. Chibotaru and A. K. Powell, J. Am. Chem. Soc., 2011, 133, 11948.

9 L. Jia, Q. Chen, Y. S. Meng, H. L. Sun and S. Gao, Chem. Commun., 2014, 50, 6052.
10 (a) Y. X. Wang, W. Shi, H. Li, Y. Song, L. Fang, Y. H. Lan, A. K. Powell, W. Wernsdorfer, L. Ungur, L. F. Chibotaru, M. R. Shen and P. Cheng, Chem. Sci., 2012, 3, 3366; (b) C. Train, R. Gheorghe, V. Krstic, L. M. Chamoreau, N. S. Ovanesyan, G. L. J. A. Rikken, M. Gruselle and M. Verdaguer, Nat. Mater., 2008, 7, 729.

11 (a) H. Zabrodsky, S. Peleg and D. Avnir, J. Am. Chem. Soc., 1992, 114, 7843; (b) M. Pinsky and D. Avnir, Inorg. Chem., 1998, 37, 5575 .

12 (a) E. L. Muetterties and L. J. Guggenberger, J. Am. Chem. Soc., 1974, 96, 1748; (b) M. G. B. Drew, Coord. Chem. Rev., 1977, 24, 179.

13 (a) J. Ruiz, A. J. Mota, A. Rodríguez-Diéguez, S. Titos, J. M. Herrera, E. Ruiz, E. Cremades, J. P. Costes and E. Colacio, Chem. Commun., 2012, 48, 7916; (b) Y. Zhu, F. Luo, Y. M. Song, H. X. Huang, G. M. Sun, X. Z. Tian, Z. Z. Yuan, Z. W. Liao, M. B. Luo, S. J. Liu, W. Y. Xu and X. F. Feng, Dalton Trans., 2012, 41, 6749; (c) Z. Chen, B. Zhao, P. Cheng, X. Q. Zhao, W. Shi and Y. Song, Inorg. Chem., 2009, 48, 3493.

14 N. Ishikawa, M. Sugita and W. Wernsdorfer, Angew. Chem., Int. Ed., 2005, 44, 2931.

15 (a) M. Jeletic, P. H. Lin, J. J. Le Roy, I. Korobkov, S. I. Gorelsky and M. Murugesu, J. Am. Chem. Soc., 2011, 133, 19286; (b) J. D. Rinehart, K. R. Meihaus and J. R. Long, J. Am. Chem. Soc., 2010, 132, 7572.

16 (a) W. Wernsdorfer, N. Aliaga-Alcalde, D. N. Hendrickson and G. Christou, Nature, 2002, 416, 406; (b) S. Hill, R. S. Edwards, N. Aliaga-Alcalde and G. Christou, Science, 2003, 302, 1015; (c) T. N. Nguyen, W. Wernsdorfer, K. A. Abboud and G. Christou, J. Am. Chem. Soc., 2011, 133, 20688.

17 Y.-Z. Zheng, Z. Zheng and X.-M. Chen, Coord. Chem. Rev., 2014, 258-259, 1. 